ERRATUM

Satoshi Awata • Hideaki Takeuchi • Masanori Kohda

\title{
The effect of body size on mating system and parental roles in a biparental cichlid fish (Julidochromis transcriptus): a preliminary laboratory experiment
}

Published online: May 31, 2006

J Ethol (2006) 24:125-132

Errors appeared in the article cited above.

"classic polyandry" should read "classical polyandry" in the 8th sentence of the 2nd paragraph in the Introduction and in the 2nd sentence of the 5th paragraph of the Mating system section of the Discussion.

S. Awata $(\bowtie) \cdot$ H. Takeuchi $\cdot$ M. Kohda

Laboratory of Animal Sociology, Department of Biology and

Geosciences, Graduate School of Science, Osaka City University,

3-3-138 Sugimoto, Sumiyoshi, Osaka, 558-8585, Japan

Tel. +81-6-66053170; Fax +81-6-66053172

e-mail: awata@sci.osaka-cu.ac.jp

Present address:

Sesoko Station, Tropical Biosphere Research Center, University of the

Ryukyus, 3422 Sesoko, Motobu, Okinawa 905-0227, Japan

The online version of the original article can be found at

http://dx.doi.org/10.1007/s10164-005-0171-5 